\title{
Persönlichkeitsstörungen sind emotionale Teilleistungsstörungen - Pro \& Kontra
}

\author{
Personality Disorders are Emotional Disorders - Pro \& Contra
}

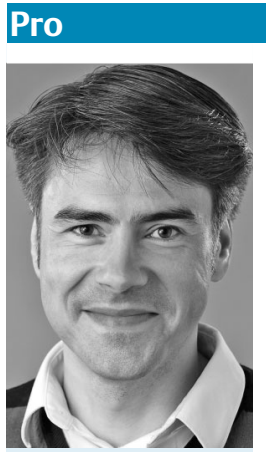

Dr. Martyn Vilain

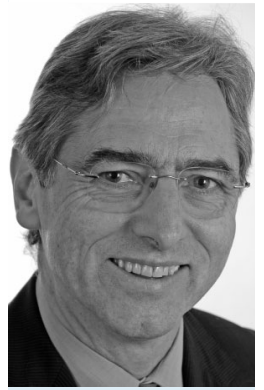

Prof. Dr. Michael Linden
Unter „Teilleistungs-

störungen“ (TLS)

wird eine Gruppe unterschiedlicher

Störungen zusammengefasst, denen gemeinsam ist, dass sie Leistungsminderungen einzelner basaler Funktionen innerhalb eines größeren funktionalen Systems beinhalten $[1,2]$. Derartige grundlegende, elementare Funktionen sind erforderlich, um höhere, komplexere Funktionen aufzubauen und auszudifferenzieren. Am besten beschrieben sind kognitive TLS wie $z$. B. Lese-Rechtschreibschwäche, Dyslexie etc. [3]. Charakteristisch ist das Zurückbleiben einzelner Fertigkeiten oder einzelner meist relativ scharf umgrenzter Aspekte von Fertigkeiten, vor dem Hintergrund einer ansonsten weitgehend unauffälligen globalen intellektuellen Leistungsfähigkeit. Es bestehen enge Beziehungen zum Konzept der minimalen zerebralen Dysfunktion [4] und es gibt Überschneidungen z.B. mit dem ADHS, bei dem neben der Leitsymptomatik der Aufmerksamkeits- und/oder Aktivitätsstörung, auch weitere TLS bestehen können, wie beispielsweise motorische Störungen oder Affektstörungen [5].

TLS des emotionalen Systems haben bislang kaum wissenschaftliche und klinische Beachtung gefunden. Es muss aber auch für emotionale Funktionen mit der Möglichkeit von TLS gerechnet werden. Erste Schritte zur Beschreibung emotionaler TLS als eigenständige Faktoren in der Erwachsenenpsychiatrie finden sich unter Bezug auf die Persönlichkeitsstörungen [3, 6-8].

Voraussetzung für die Beschreibung emotionaler TLS ist eine Systematik, die erlaubt verschiedene Dimensionen der Emotionalität und der Emotionalitätsstörungen zu beschreiben. Hier kommt dem psychopathologischen Befund eine zentrale Bedeutung $\mathrm{zu}$, in dem emotionale TLS durch die psychopathologische Beschreibung von Affektstörungen, mit der Unterscheidung zwischen Affektqualitäten und Affektregulation, dargestellt werden können. Affektqualitäten sind normale oder pathologische Emotionszustände, wie z. B. Freude, Trauer oder Angst. Die Affektregulation umfasst die (a) Affektadäquatheit, (b) Affektintensität und (c) Affektauslenkbarkeit bzw. -stabilität.

Gesunden Menschen ist es möglich, situations- und kontextabhängig unterschiedliche Affekte zu produzieren. Unter Affektadäquatheit versteht man die kontextadäquate Produktion bzw. Auswahl eines Affektes. Eine Störung in der Affektadäquatheit kann darin bestehen, dass immer der gleiche Affekt produziert wird. Dies zeigt sich z.B. wenn situationsübergreifend, oder ausgelöst durch für die Störung typische Situationen, immer der gleiche Dominanzaffekt gezeigt wird, wie beispielsweise Misstrauen bei der paranoiden Persönlichkeit. Dabei ist der relativ autonome, nicht bewusste Vorgang der Ausbildung einer Emotionsqualität gemeint.

Störungen der Affektintensität äußern sich in einer verstärkten oder auch abgeflachten Emotionsfülle und betreffen das Emotionserleben und den Emotionsausdruck. Es kann die Intensität der einzelnen Emotion im subjektiven Erleben des Patienten oder im Ausdruck der Emotion (von außen beobachtbar) betroffen sein. Eine verstärkte Affektexpressivität findet sich beispielsweise bei histrionischen Persönlichkeitsstörungen. Die hierbei gezeigten Emotionen können ihrer Qualität nach oft durchaus als situationsadäquat angesehen werden, die Intensität der zum Ausdruck gebrachten Emotion erscheint jedoch zu stark. Das Gegenstück ist ein reduzierter Affektausdruck, wie er z.B. bei schizoiden Persönlichkeiten beobachtet werden kann. Die Emotionen des Betroffenen sind ebenfalls situationsangemessen, werden vom Gegenüber jedoch nicht als voller, holothymer Affekt wahrgenommen und z.T. auch von den Betroffenen selbst als wenig spürbar beschrieben.

Die Affektstabilität beschreibt, wie stark eine Emotion durch Außenstimulation anzustoßen ist. Da Emotionen wesentlich der zwischenmenschlichen Interaktion dienen, müssen sie auch von außen anregbar sein und sich in der Interaktion gegenseitig beeinflussen. Kommt es in Relation zum Anlass in unterschiedlichen Situationen und im Bezug auf verschiedene Affekte, zu unverhältnismäßig starken Affektauslenkungen, dann wird dies als Affektinkontinenz bezeichnet. Das Gegenteil ist die Affektarmut bzw. die emotionale Kälte und der Empathiemangel. Hier reagieren Betroffene emotional nicht auf äußere Ereignisse oder die Emotionalität des Gegenübers. Ein normaler Affekt hat zudem eine Stetigkeit über Zeitstrecken von mehreren Minuten bis Stunden. Im Gegensatz dazu kommt es bei der Affektlabilität zu einem sehr schnellen Wechsel von einer Emotionsqualität in eine andere, wie dies typisch ist für Borderline-Störungen.

Störungen der Affektqualität und -regulation können im Kontext unterschiedlichster Erkrankungen beobachtet werden. Wenn sie jedoch als weitgehend singuläre und eng umschriebene Störung auftreten, bei gleichzeitig ansonsten normalen kognitiven und psychischen Funktionen, dann können sie als emotionale TLS verstanden werden. Sucht man nach Kriterien, die spezifisch für einzelne Persönlichkeitsstörungen sind, dann bleibt ergänzend zu eher unspezifischen Kriterien (lang anhaltend, situationsübergreifend, mit einem inadäquaten Interaktionsverhalten und Beeinträchtigung der Umweltadaptation) letztlich nur die Störung der Affektivität in Verbindung mit typischen kognitiven Mustern (z.B.: paranoide PS: „Alle sind gegen mich“, abhängige PS: „Alleine schaffe ich es nicht", histrionische PS: „Ich muss Eindruck machen“).

Einerseits führen die beschrieben Störungen in der Emotionalität zu weitreichenden Interaktions- und Anpassungsstörungen. Andererseits können gestörte Emotionen beim Betroffenen selbst zu situativen Fehldeutungen führen. Was wir fühlen, was wir denken und wahrnehmen, hängt wesentlich von den Emotionen ab, die wir in einer bestimmten Situation haben. Es kommt zu einem geschlossenen Kreis, einem „Circulus vitiosus“ mit „Self fullfilling prophecy“. Eine weitere Konsequenz ist, dass der Patient auf dem Hintergrund solcher Erfahrungen korrespondie- 
rende kognitive Schemata ausbildet. $\mathrm{Zu}-$ sammenfassend können also sowohl die ersten Eindrücke, die man von Menschen mit Persönlichkeitsstörungen gewinnt, wie auch die typischen Anpassungsstörungen, Interaktionsstörungen und dysfunktionalen Kognitionen als unmittelbare Folge einer gestörten Emotionalität bzw. einer persistierenden emotionalen TLS verstanden werden.

Über die Ursachen emotionaler TLS wie auch der Persönlichkeitsstörungen insgesamt kann nur spekuliert werden. Sie können theoretisch eine Folge angeborener oder erworbener Entwicklungsstörungen oder auch von neuro- oder psychotraumatischen Schädigungen sein. Was auch immer die jeweilige Ursache sein mag, so verdichten sich zunehmend die Hinweise auf neurobiologische Auffälligkeiten bei Patienten mit Persönlichkeitsstörungen, sodass strukturell und funktional veränderte neurophysiologisch bedingte Reaktionsmuster angenommen werden müssen, die basaler sind als erlernte Kognitionen oder soziale Kompetenzen. Die neurologisch erkennbaren Auffälligkeiten betreffen Strukturen und Funktionen des limbischen und retikulären Systems und zeigen sich in veränderten emotionalen Reaktionen auf positive wie negative Stimulationen unterschiedlichster Art $[9,10]$.
Das Konzept der emotionalen TLS ermöglicht ein anderes Verständnis von Persönlichkeitsstörungen, welches sich zukünftig auch in diagnostischen und therapeutischen Überlegungen niederschlägt.

\section{Literatur}

1 Naggl M. „Teilleistungsstörungen“ - die Entwicklung eines Konzepts. Frühförderung interdisziplinär 1994; 13: 1-9

2 Pritzel M, Schwandt B. Neurobiologische Korrelate von Störungen der Gehirn- und Verhaltensentwicklung. In: Markowitsch $\mathrm{HJ}$ ed. Klinische Neuropsychologie. Göttingen: Hogrefe; 1997: 1-90

3 Vellutino $F$, Fletcher J, Snowling $M$ et al. Specific reading disability (dyslexia): what have we learned in the past four decades? Journal of Child Psychology and Psychiatry 2004; 45: 2-40

4 Esser G, Schmidt M. Minimale Cerebrale Dysfunktion - Leerformel oder Syndrom? Empirische Untersuchung zur Bedeutung eines zentralen Konzepts in der Kinderpsychiatrie. Stuttgart: Enke; 1987

5 Barkley R. Attention-Deficit Hyperactivity Disorder. A Handbook for Diagnosis and Treatment. New York: Guilford; 2006

6 Steckel K. Persönlichkeitsmerkmale erwachsener ADHS-Patienten: Veränderungen unter der Behandlung mit Methylphenidat. Medizinische Dissertation an der Ruhr Universität. Bochum: 2005

7 Winkler M, Rossi P. Borderline-Persönlichkeitsstörung und Aufmerksamkeitsdefizit-/ Hyperaktivitätsstörung bei Erwachsenen. Persönlichkeitsstörungen 2001; 5: 39-48
8 Linden M. Minimal emotional dysfunctions (MED) in personality disorders. European Journal of Psychiatry 2006: 325-332

9 Herpertz S, Dietrich T, Wenning B et al. Evidence of abnormal amygdala functioning in borderline personality disorder: a functional MRI study. Biological Psychiatry 2001; 50: $292-298$

10 DeKloet ER, Siburg RM, Helmerhorst FM et al. Stress, genes and the mechanism of programming the brain for later life. Neurosci Biobehav Rev 2004; 29: $271-281$

\section{Korrespondenzadressen \\ Dr. Martyn Vilain \\ Prof. Dr. Michael Linden}

Forschungsgruppe Psychosomatische

Rehabilitation an der Charité - Universitätsmedizin Berlin und Abteilung für Verhaltenstherapie und

Psychosomatik am Reha-Zentrum Seehof der

Deutschen Rentenversicherung Bund

Lichterfelder Allee 55

14513 Teltow

vilain@zppb.de

michael.linden@charite.de

Bibliografie

DOI http://dx.doi.org/

10.1055/s-0032-1305092

Psychiat Prax 2012; 39: 259-260

(c) Georg Thieme Verlag KG

Stuttgart · New York

ISSN 0303-4259 